\title{
Space VLBI Assistance Software-A New Version
}

\author{
S. Frey, I. Fejes, G. Heitler, I. Noszticzius, \& Z. Paragi \\ FÖMI Satellite Geodetic Observatory, Penc, Hungary
}

Abstract. A new version of the Space VLBI Assistance Software (SPAS) developed at the FÖMI Satellite Geodetic Observatory (Penc, Hungary) is described. SPAS version 2.0 will be a useful software tool for proposal preparation in response to the second Announcement of Opportunity of the VSOP satellite.

\section{Introduction}

The Satellite Geodetic Observatory of the Institute of Geodesy, Cartography and Remote Sensing (FÖMI), Hungary has been involved in space VLBI related research since the mid-eighties. This research concentrates on both astrophysical and geodetic applications of the new observing technique. One of the main activities was to develop a user assistance software in order to help the user community to prepare observing proposals in response to the Announcement of Opportunity (AO) of space VLBI satellites. The Space VLBI Assistance Software (SPAS) is being developed since 1993 (Fejes et al. 1994, Noszticzius 1994, Frey 1995) using the software engineering standards of the European Space Agency (ESA 1991). The first version of SPAS was released in June 1995. Together with other two user support software packages (Murphy 1995), SPAS was used for VSOP proposal preparation worldwide simulating space VLBI experiments by checking satellite constraints, ground VLBI and tracking network availability and assessing the expected data quality. Here we give a summary of the capabilities of SPAS and describe the new and improved features incorporated in the second program version. SPAS version 2.0 is intended to be released before the next VSOP AO expected for the end of 1997.

\section{General Description of SPAS}

SPAS is a PC-based software package with windows-like graphical user interface. The program runs on IBM PC compatible computers without any extreme hardware requirement. The executable programs, the necessary input data files and the user manual are distributed via Internet or floppy disks. SPAS is easy to install. The mouse and/or keyboard driven menu system and icons make its use convenient.

The main purpose of the software is to help potential space VLBI proposers to simulate and approximately "schedule" space VLBI experiments. The latest version of SPAS has 16 different operations which visualize the geometry of the ground VLBI network and space VLBI satellite(s), check observing and technical constraints of the satellites and ground based equipment, estimate the sensitivity of the system, check the $(u, v)$ coverage for given radio sources, etc. The different operations, however, use a common data base for radio sources, satellites, ground VLBI and tracking stations. Input parameters can be set up in a very similar way for all of these operations. There is a data base handling program included in the SPAS package to view and edit catalogue files. Default 
catalogues are provided with SPAS. Most of the operations can handle 20 ground VLBI stations, 20 satellite tracking stations and 2 space VLBI satellites in a simulated experiment at the same time. The checking of major satellite pointing restrictions is implemented in the program. The satellite on-board constraints are included in the default data base for both VSOP and Radioastron.

\section{New Program Version}

Further developments in SPAS version 2.0 are based on the experience gained during the first VSOP proposal period in 1995. Three new operations are implemented. Two of them provide a more convenient way to plot $(u, v)$ coverage as a funcion of time or source position. $(u, v)$ coverage information appeared to be one of the most important factors in designing and proposing space VLBI experiment for VSOP satellite.

Other changes are based on suggestions received from many of the program users. Data base modifications include the extension of default radio source catalogue with more bright sources and more flux density values, and the possibility of marking e.g. calibrator sources. The format of the catalogue files, however, remains compatible with that of the previous program versions. The updated VSOP orbital elements and on-board receiver characteristics will be entered in the satellite catalogue just before the release. Program handling becomes more convenient as configuration parameters (e.g. printer, working directories) can be changed from within the SPAS graphical user interface. Some of the "old" subprograms have advanced features in the 2.0 version. The SPAS User Manual is also revised and updated. It will be included in the package in PostScript format as before.

For up-to-date information on the latest SPAS developments and the availability of the package one can visit the SPAS home page on the WWW:

$$
\text { http://www.sgo.fomi.hu/vlbi/spas.htm }
$$

The program can be downloaded from the Satellite Geodetic Observatory's ftp server in Penc, Hungary:

$$
\text { ftp.sgo.fomi.hu or 193.224.183.66, directory /spas/exe }
$$

Acknowledgments. The software development was supported by the EU (contract no. CHGECT 920011), the Hungarian Space Office and the US-Hungary Science and Technology Program.

\section{References}

Fejes I., et al. 1994. in VLBI Technology: Progress and Future Observational Possibilities, eds. Sasao T., Manabe S., Kameya O. \& Inoue M. (Tokyo: Terra), 59-69.

Frey S. 1995. YERAC.

Murphy D. W. 1995. BAAS, 27, 851.

Noszticzius I. 1994. in Proc. 2nd EVN/JIVE Symposium, eds. Kus A. J., Schilizzi R. T., Borkowski K. M. \& Gurvits L. I. (Dwingeloo: JIVE), 25-31. 